\title{
Global stability for a discrete SIR epidemic model with delay in the general incidence function
}

\author{
Aboudramane Guiro ${ }^{1 *}$, Dramane Ouedraogo ${ }^{1}$ and Harouna Ouedraogo ${ }^{1}$ \\ ${ }^{1}$ Département de Mathématiques, UFR des Sciences et Techniques, Université Nazi Boni, Bobo-Dioulasso, \& LAboratoire de \\ Mathématique et d'Informatique (LAMI), B.P. 1091 Bobo-Dioulasso, Burkina Faso \\ *Corresponding author E-mail: abouguiro@yahoo.fr, abouguiro@gmail.com
}

\begin{abstract}
In this paper, we construct a backward difference scheme for a class of general SIR epidemic model with general incidence function $f$. We use the step size $h>0$, for the discretization. The dynamical properties are investigated (positivity and the boundedness of solution). By constructing the Lyapunov function, under the conditions that function $f$ satisfies some assumptions. The global stabilities of equilibria are obtained. If the basic reproduction number $R_{0} \leq 1$, the disease-free equilibrium is globally asymptotically stable. If $R_{0}>1$, the endemic equilibrium is globally asymptotically stable.
\end{abstract}

Keywords: discrete SIR epidemic model, general incidence; Lyapunov function; backward difference scheme; local stability; global stability.

\section{Introduction}

In the theory of epidemic dynamical models there are two kinds of mathematical models, the continuous-time models described by differential equations and the discrete-time models described by difference equations.

Nowadays, in order to study the continuous time SIR and SIRS epidemic models, many various discrete dynamical model have been constructed and then, dynamical properties have been considered in many papers such as $([9,5,11,12,13])$. The fact that the epidemiological data are usually collected in discrete time units, such as daily, weekly or monthly, makes the discrete model a natural choice to describe a disease transmission. The result of the discrete model can also be compared with the actual data directly. The discrete model also exhibits rich dynamical behavior, though it is more complicated than the corresponding continuous model. However, the study of the discrete epidemic models is comparatively few due to the complicates expressions and the difficulties in the dynamical analysis. In this paper, we discretize the continuous-time model studied in [6], by using the backward difference scheme with time step size $h$. The SIR model under consideration here use a general incidence function with delay $f\left(S, I_{\tau}\right)$ and generalize a $S I R$ model studied in [2] with a particular incidence function $\frac{\beta S I_{\tau}}{1+\alpha I_{\tau}}$

In [2], a detailed analysis of the current model is presented. It is shown that when the basic reproduction number $R_{0} \leq 1$, then the diseasefree equilibrium is globally asymptotically stable and when the $R_{0}>1$, then the endemic equilibrium is globally asymptotically stable, without any further conditions on the parameters. In this paper, we construct a backward difference scheme for a class of continuous-time SIR models with general nonlinear incidence function with delay. We study the dynamical properties, especially the global stability of the disease-free equilibrium and endemic equilibrium for this discrete model. Primary, we study the basic properties of the model, such as, the positivity and the boundedness of solutions. Furthermore, we construct discrete type of Lyapunov functions and use stability theory of difference equations to establish the global stability of equilibria.

The paper is organized as followed, in second section we give the discrete mathematical model, the basic reproduction number $R_{0}$ and the existence and uniqueness of disease-free equilibrium and endemic equilibrium. Thirdly, we study the positivity and the boundedness of solution. At fourth, we study the stability of disease-free equilibrium, when $R_{0} \leq 1$. In section 5 , we study the global stability of unique endemic equilibrium when $R_{0}>1$. In the section 6 , we give a particular incidence function used for our numerical simulation and the numerical results. We end with some remarks a conclusion. 


\section{Discrete mathematical mode}

In this section, we study a discrete SIR mathematical model. In fact, we considere the following continuous model from [2] with a general nonlinear incidence function given by:

$$
\left\{\begin{array}{l}
\dot{S}=B-\mu_{1} S-f\left(S, I_{\tau}\right), \\
\dot{I}=f\left(S, I_{\tau}\right)-\left(\mu_{2}+\gamma\right) I, \\
\dot{R}=\gamma I-\mu_{3} R .
\end{array}\right.
$$

Where

$\mu_{i}, i=1,2,3$ denote the death rate of the different class,

$S$ is the size of the susceptible individus,

$I$ is the size of the infected individus,

$R$ is the size of the individus recovered, $B$ is the recruitment rate of susceptible hots,

$\gamma$ is the treatment rate of infected,

$f$ is a general incidence function, which must satisfies some conditions.

We make the following hypothesis

H1: $f$ is non-negative $C^{1}$ function on the non-negative orthant.

H2: for all $(S, I) \in \mathbb{R}_{+}^{2}, f(S, 0)=f(0, I)=0$.

Let us denote by $f_{1}$ and $f_{2}$ the partial derivatives of $f$ with respect to the first and to the second variable.

We assume that system (1) holds with initial conditions

$$
S(0) \in \mathbb{R}_{+} \text {and } I(\theta)=\phi(\theta) \text { for } \theta \in[-j, 0] ; 0 \leq j \leq n-1 .
$$

Let us denote by $\phi \in \mathscr{C}=C\left([-j, 0], \mathbb{R}_{+}\right)$, the space of continuous functions from $[-j, 0]$ to $\mathbb{R}_{+}$.

The disease-free equilibrium of system (1) is given by,

$$
E_{0}=\left(S^{0}, I^{0}, R^{0}\right)=\left(\frac{B}{\mu_{1}}, 0,0\right)
$$

and the endemic equilibrium by:

Also, the basic reproduction number is

$$
E^{*}=\left(S^{*}, I^{*}, R^{*}\right)
$$

$$
R_{0}=\frac{f_{2}\left(S^{0}, 0\right)}{\mu_{2}+\gamma}
$$

Remark 2.1: The basic reproduction number evaluates the average number of new infections generated by a single infected individual in a completely susceptible population. We can summarize the stability of the equilibria by the following theorem:

Theorem 2.1: The following statements hold.

(i) If $R_{0} \leq 1$. Then the disease-free equilibrium $E_{0}$ of (1) is globally asymptotically stable.

(ii) Let assume $R_{0}>1$. Then the endemic equilibrium $E^{*}$ of (1) is globally asymptotically stable.

Now, we use the backward difference scheme to discretize model (1). Let $h>0$ be the time step size.

Since

$\frac{d S(t)}{d t}=\lim _{h \rightarrow 0} \frac{S(t+h)-S(t)}{h}, \quad \frac{d I(t)}{d t}=\lim _{h \rightarrow 0} \frac{I(t+h)-I(t)}{h}$,

and $\frac{d R(t)}{d t}=\lim _{h \rightarrow 0} \frac{R(t+h)-R(t)}{h}$,

we obtain

$\lim _{h \longrightarrow 0}\left(B-\mu_{1} S(t+h)-f\left(S(t+h), I^{j}(t+h)\right)\right)=B-\mu_{1} S(t)-f\left(S(t), I_{\tau}(t)\right)$

$\lim _{h \longrightarrow 0}\left(f\left(S(t+h), I^{j}(t+h)\right)-\left(\mu_{2}+\gamma\right) I(t+h)\right)=f\left(S(t), I_{\tau}(t)\right)-\left(\mu_{2}+\gamma\right) I(t)$

and

$\lim _{h \rightarrow 0} \gamma I(t+h)-\mu_{3} R(t+h)=\gamma I(t)-\mu_{3} R(t)$.

Therefore, we can derive from the continuous model (1), the discrete one for any step size $h>0$, by using a variation of backward Euler method:

$$
\left\{\begin{array}{l}
\frac{S(t+h)-S(t)}{h}=B-\mu_{1} S(t+h)-f\left(S(t+h), I^{j}(t+h)\right) \\
\frac{I(t+h)-I(t)}{h}=f\left(S(t+h), I^{j}(t+h)\right)-\left(\mu_{2}+\gamma\right) I(t+h) \\
\frac{R(t+h)-R(t)}{h}=\gamma I(t+h)-\mu_{3} R(t+h) .
\end{array}\right.
$$


Let us denote by

$t=n, t+h=n+1, S(t)=S_{n}, I(t)=I_{n}, R(t)=R_{n}, S(t+h)=S_{n+1}, I(t+h)=I_{n+1}$, and $R(t+h)=R_{n+1}$.

From system (2) we obtain the following discrete SIR epidemic model with general nonlinear incidence function:

$$
\left\{\begin{array}{l}
S_{n+1}-S_{n}=h\left[B-\mu_{1} S_{n+1}-f\left(S_{n+1}, I_{n+1}^{j}\right)\right] \\
I_{n+1}-I_{n}=h\left[f\left(S_{n+1}, I_{n+1}^{j}\right)-\left(\mu_{2}+\gamma\right) I_{n+1}\right] \\
R_{n+1}-R_{n}=h\left[\gamma I_{n+1}-\mu_{3} R_{n+1}\right] .
\end{array}\right.
$$

Where $B, \gamma$ and $\mu_{i}, i=1,2,3$ are some positive constants, similar to those in the continuous system (1). Since $R_{n}$ does not appear in the first and the second equation of system (3), it is sufficient to analyze the behavior of $S_{n}$ and $I_{n}$.

System (3) always admits a disease-free equilibrium

$$
E_{0}=\left(\frac{B}{\mu_{1}}, 0,0\right)
$$

and an endemic equilibrium

$$
E^{*}=\left(S^{*}, I^{*}, R^{*}\right)
$$

where

$$
S^{*}=\frac{\beta-\left(\mu_{2}+\gamma\right) I^{*}}{\mu_{1}} \text { and } R^{*}=\frac{\gamma}{\mu_{3}} I^{*}
$$

Let also make these hypothesis:

H3: $f\left(S_{n}, I_{n}^{j}\right) \leq f_{2}\left(S^{0}, 0\right) I_{n}$ for all $n \geq 0$ and $j \leq n$.

H4: for all $n \geq 0, \quad \frac{I_{n+1}}{I^{*}} \leq \frac{S_{n+1}}{S^{*}} \leq \frac{f\left(S_{n+1}, I_{n+1}^{j}\right)}{f\left(S^{*}, I^{*}\right)}$.

Remark 2.2: The assumption $\mathbf{H 4}$ is used to prove that the difference of the Lyapunov function $V_{n+1}-V_{n} \leq 0$, in addition, this assumption is also used to show that $S_{n}>0 \forall n \in \mathbb{N}$.

\section{Basic properties}

We consider that the initial conditions of system (3):

$S(0)>0, \quad I(0)>0$ and $R(0)>0$.

Proposition 3.1: Let $\left(S_{n}, I_{n}, R_{n}\right)$ be the solution of system (3), with initial conditions (8). Then $S_{n}>0, I_{n}>0$ and $R_{n}>0$ for all $n$. Proof. To prove this proposition, we use the system (3).

Assume that $S_{n}>0, I_{n}>0$ and $R_{n}>0$.

From the second equation of (3) we have,

$$
\left(1+h\left(\mu_{2}+\gamma\right)\right) I_{n+1}=I_{n}+h f\left(S_{n+1}, I_{n+1}^{j}\right),
$$

since $I_{n}>0$ and $f$ a non-negative function, we have $I_{n+1}>0$ for all step size $h>0$.

From the last equation of system (3) we have,

$$
\left(1+h \mu_{3}\right) R_{n+1}=R_{n}+h \gamma I_{n+1},
$$

since $R_{n}>0$ and $I_{n+1}>0$; then, we have $R_{n+1}>0$ for all step size $h>0$.

For the positiveness of $S_{n+1}$ we use the assumption $\mathbf{H 4}$ and then, we get $\left(S_{n}, I_{n}, R_{n}\right) \in \mathbb{R}_{+}^{* 3}$ for all $n \geq 0$

Proposition 3.2: Any solution $\left(S_{n}, I_{n}, R_{n}\right)$ of system (3), with initial condition (4) satisfies

$$
\lim _{n \rightarrow+\infty} \sup \left(S_{n}+I_{n}+R_{n}\right) \leq \frac{B}{\mu}
$$

with $\mu=\min \left\{\mu_{1}, \mu_{2}, \mu_{3}\right\}$. Proof. Let us note $N_{n+1}=S_{n+1}+I_{n+1}+R_{n+1}$ and $N_{n}=S_{n}+I_{n}+R_{n}$. By adding the different equation of system (3) we have,

$$
\begin{aligned}
N_{n+1}-N_{n} & =h\left[B-\mu_{1} S_{n+1}-\mu_{2} I_{n+1}-\mu_{3} R_{n+1}\right] \\
& \leq h\left[B-\mu\left(S_{n+1}+I_{n+1}+R_{n+1}\right)\right] \\
& \leq h\left[B-\mu N_{n+1}\right] \\
& \Longrightarrow(1+h \mu) N_{n+1}-N_{n} \leq h B,
\end{aligned}
$$

so

$$
\begin{gathered}
\lim _{n \rightarrow+\infty} \sup \left((1+h \mu) N_{n+1}-N_{n}\right)=\lim _{n \rightarrow+\infty} \sup h \mu N_{n+1} \\
\Longrightarrow \lim _{n \rightarrow+\infty} \sup N_{n+1} \leq \frac{B}{\mu} .
\end{gathered}
$$

Hence we have

$$
\lim _{n \longrightarrow+\infty} \sup \left(S_{n}+I_{n}+R_{n}\right) \leq \frac{B}{\mu}
$$

with $\mu=\min \left\{\mu_{1}, \mu_{2}, \mu_{3}\right\}$.

Theorem 3.1: 
(i) If $R_{0} \leq 1$, then model (3) has only a unique disease-free equilibrium

$$
E_{0}=\left(S^{0}, 0,0\right) .
$$

(ii) If $R_{0}>1$, then model (3) has a unique endemic equilibrium

$$
E^{*}=\left(S^{*}, I^{*}, R^{*}\right) .
$$

Proof. Any equilibrium $E=(S, I, R)$ satisfies

$$
\left\{\begin{array}{l}
B-\mu_{1} S-f(S, I)=0 \\
f(S, I)-\left(\mu_{2}+\gamma\right) I=0 \\
\gamma I-\mu_{3} R=0
\end{array}\right.
$$

By using the second equation of (5) we obtain:

$$
\frac{f(S, I)}{I}=\left(\mu_{2}+\gamma\right)
$$

Now let us consider the function $\varphi$ defined by,

$$
\varphi(I)=\frac{f\left(S^{0}-\frac{\mu_{2}+\gamma}{\mu_{1}} I, I\right)}{I}-\left(\mu_{2}+\gamma\right) .
$$

Hence, we have

$$
\begin{aligned}
\lim _{I \rightarrow 0^{+}} \varphi(I) & =\frac{\partial f}{\partial I}\left(\left(S^{0}, 0\right)\right)-\left(\mu_{2}+\gamma\right) \\
& =\left(\mu_{2}+\gamma\right)\left(\frac{f_{2}\left(S^{0}, 0\right)}{\mu_{2}+\gamma}-1\right) \\
& =\left(\mu_{2}+\gamma\right)\left(R_{0}-1\right),
\end{aligned}
$$

and in the other hand

$$
\varphi(\bar{I})=-\left(\mu_{2}+\gamma\right), \text { where } \bar{I}=\frac{\mu_{1} S^{0}}{\mu_{2}+\gamma} .
$$

When $R_{0} \leq 1$, we have $\lim _{I \rightarrow 0^{+}} \varphi(I) \leq 0$. Consequently, there is not any $I^{*}>0$ such that $\varphi\left(I^{*}\right)=0$. Therefore, model (3) has a unique disease-free equilibrium $E_{0}$. When $R_{0}>1$, we have $\lim _{I \rightarrow 0^{+}} \varphi(I)>0$. Therefore, there exists a unique $\left.I^{*} \in\right] 0 ; \bar{I}\left[\right.$ such that $\varphi\left(I^{*}\right)=0$.

Furthermore, we have $S^{*}=S^{0}-\frac{\mu_{2}+\gamma}{\mu_{1}} I^{*}>0$ and $R^{*}=\frac{\gamma}{\mu_{3}} I^{*}>0$ and this implies that model (3) has unique endemic equilibrium $E^{*}=\left(S^{*}, I^{*}, R^{*}\right)$.

Reamark 3.1: The space $K=\mathbb{R}_{+} \times \mathscr{C}$ is positively invariant and attracting domain for system (3).

\section{Stability of the disease-free equilibrium}

\subsection{Local stability of the disease-free equilibrium}

In this subsection, we will study the stability of the disease-free equilibrium $E_{0}=\left(\frac{B}{\mu_{1}}, 0,0\right)$.

Theorem 4.1: When $R_{0} \leq 1$, then disease-free equilibrium $E_{0}$ of model (3) is locally asymptotically stable.

Proof. By computing the linearization system of model (3) at equilibrium $E_{0}$, we have

$$
\left\{\begin{array}{l}
S_{n+1}-S_{n}=h\left[-\mu_{1}-f_{1}\left(S^{0}, 0\right)\right] S_{n+1}-h f_{2}\left(S^{0}, 0\right) I_{n+1} \\
I_{n+1}-I_{n}=h f_{1}\left(S^{0}, 0\right) S_{n+1}+h\left[f_{2}\left(S^{0}, 0\right)-\left(\mu_{2}+\gamma\right)\right] I_{n+1} \\
R_{n+1}-R_{n}=h \gamma I_{n+1}-h \mu_{3} R_{n+1} .
\end{array}\right.
$$

The matrix $A$ associate of system (6) is given by

$$
A=\left(\begin{array}{ccc}
1+h\left[\mu_{1}+f_{1}\left(S^{0}, 0\right)\right] & h f_{2}\left(S^{0}, 0\right) & 0 \\
-h f_{1}\left(S^{0}, 0\right) & 1-h\left[f_{2}-\left(\mu_{2}+\gamma\right)\right] & 0 \\
0 & -h \gamma & 1+h \mu_{3}
\end{array}\right)
$$

The system (6) can be rewrite by

with $X_{n}=\left(S_{n}, I_{n}, R_{n}\right)^{t}$.

$$
X_{n+1}=A^{-1} X_{n}
$$

If all eigenvalues $\lambda$ of $A$ satisfy $|\lambda|>1$, then eigenvalues $\sigma$ of $A^{-1}$ will satisfy $|\sigma|<1$. 
The characteristic polynomial $P(X)$ associated to $A$ is:

$$
\begin{aligned}
P(X)= & \operatorname{det}(A-X I) \\
= & \left(1+h \mu_{3}-X\right)\left[( 1 + h \mu _ { 1 } + h f _ { 1 } ( S ^ { 0 } , 0 ) - X ) \left(1-h f_{2}\left(S^{0}, 0\right)\right.\right. \\
& \left.\left.+h\left(\mu_{2}+\gamma\right)-X\right)+h^{2} f_{1}\left(S^{0}, 0\right) f_{2}\left(S^{0}, 0\right)\right] .
\end{aligned}
$$

Let $\lambda$ be a root of $P(X)$, then

$\left(1+h \mu_{3}-\lambda\right)=0$

or

$\left(1+h \mu_{1}+h f_{1}\left(S^{0}, 0\right)-\lambda\right)\left(1-h f_{2}\left(S^{0}, 0\right)+h\left(\mu_{2}+\gamma\right)-\lambda+h^{2} f_{1}\left(S^{0}, 0\right) f_{2}\left(S^{0}, 0\right)=0\right.$.

The root $\lambda=1+h \mu_{3}$ is strictly greater than one.

Now we prove that the roots of the polynomial equation

$\left(1+h \mu_{1}+h f_{1}\left(S^{0}, 0\right)-\lambda\right)\left(1-h f_{2}\left(S^{0}, 0\right)+h\left(\mu_{2}+\gamma\right)-\lambda\right)+h^{2} f_{1}\left(S^{0}, 0\right) f_{2}\left(S^{0}, 0\right)=0$

are also strictly greater than one.

For this, let us suppose that equation (8) have root $\lambda_{1}=|\lambda|<1$.

Thus, we have

$\left(1+h \mu_{1}+h f_{1}\left(S^{0}, 0\right)-\lambda_{1}\right)\left(1-h f_{2}\left(S^{0}, 0\right)+h\left(\mu_{2}+\gamma\right)-\lambda_{1}\right)+h^{2} f_{1}\left(S^{0}, 0\right) f_{2}\left(S^{0}, 0\right)=0$.

So from (9), we have:

$\left(1+h \mu_{1}+h f_{1}\left(S^{0}, 0\right)-\lambda_{1}\right)\left(1-h f_{2}\left(S^{0}, 0\right)+h\left(\mu_{2}+\gamma\right)-\lambda_{1}\right)=-h^{2} f_{1}\left(S^{0}, 0\right) f_{2}\left(S^{0}, 0\right)$.

By using the second member of (10) and the fact that $R_{0} \leq 1$, we get:

$$
\begin{aligned}
-h^{2} f_{1}\left(S^{0}, 0\right) f_{2}\left(S^{0}, 0\right) & =\left(h f_{1}\left(S^{0}, 0\right)\right)\left(-h f_{2}\left(S^{0}, 0\right)\right) \\
& \leq\left(h \mu_{1}+h f_{1}\right)\left(-h\left[f_{2}\left(S^{0}, 0\right)-\left(\mu_{2}+\gamma\right)\right]\right) \\
& <\left(1+h\left(\mu_{1}+f_{1}\right)-\lambda_{1}\right)\left(1-h\left[f_{2}\left(S^{0}, 0\right)-\left(\mu_{2}+\gamma\right)\right]-\lambda_{1}\right) .
\end{aligned}
$$

Hence equation (10) cannot have roots. Hence, $E_{0}$ is locally asymptotically stable according to Theorem 2 in [10].

\subsection{Global stability of the disease-free equilibrium}

Theorem 4.2: The disease-free equilibrium is globally asymptotically stable in $K$ whenever $R_{0} \leq 1$. Proof. In this proof we used the comparison theorem [8] and the assumption $H 3$. By using the equation of the infectious class in (3) and the assumption $H 3$ above, we have:

$I_{n}=\left[1+h\left(\mu_{2}+\gamma\right)\right] I_{n+1}-h f\left(S_{n+1}, I_{n+1}^{j}\right)$,

so

$$
I_{n} \geq\left(1+h\left[\left(\mu_{2}+\gamma\right)-f_{2}\left(S^{0}, 0\right)\right]\right) I_{n+1},
$$

and

$$
M^{-1} I_{n} \geq I_{n+1} \text { with } M=1+h\left[\left(\mu_{2}+\gamma\right)-f_{2}\left(S^{0}, 0\right)\right] .
$$

By using the fact that $R_{0} \leq 1$, we have $\left(\mu_{2}+\gamma\right)-f_{2}\left(S^{0}, 0\right) \geq 0$. So the constant $M$ is greater than one. We conclude that the linearized equation (11) is stable whenever $R_{0} \leq 1$. By a standard comparison theorem [8], $I_{n} \rightarrow 0$ as $n \rightarrow \infty$ for equation (11) and substituting $I_{n}=0$ in system (3), we get $S_{n} \rightarrow S^{0}, I_{n} \rightarrow 0$ as $n \rightarrow+\infty$. Thus, $\left(S_{n}, I_{n}\right) \rightarrow\left(S^{0}, 0\right)$ as $n \rightarrow+\infty$ for system (3); when $R_{0} \leq 1$. Therefore, $E_{0}$ is globally asymptotically stable if $R_{0} \leq 1$.

\section{Global stability of the endemic equilibrium}

In this section, we study the global stability of the endemic equilibrium given by $E^{*}=\left(S^{*}, I^{*}, R^{*}\right)$. Theorem 5.1: When $R_{0}>1$, the endemic equilibrium point $E^{*}$ is globally asymptotically stable. Proof. By using the first and second equation of (3), at equilibrium point $E^{*}$, we have:

$$
B=\mu_{1} S^{*}+f\left(S^{*}, I^{*}\right)
$$

and

$$
f\left(S^{*}, I^{*}\right)=\left(\mu_{2}+\gamma\right) I^{*}
$$

which can be used for the following computation.

Let

$$
g(x)=x-1-\ln x
$$


and

Let us consider

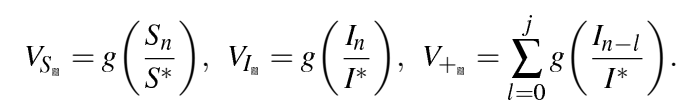

Thus

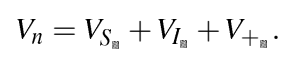

Let us calculate $V_{S_{\mathrm{a}+1}}-V_{S_{\mathrm{m}}}$.

$$
V_{n+1}-V_{n}=V_{S_{\mathrm{w}+1}}-V_{S_{\mathrm{w}}}+V_{I_{\mathrm{w}+1}}-V_{I_{\mathrm{m}}}+V_{+_{\mathrm{w}+1}}-V_{+_{\mathrm{m}}} .
$$

For this, we use the mean value theorem and we suppose that $S_{n} \leq S_{n+1}$, the computation is the same when $S_{n+1} \leq S_{n}$.

$$
\begin{aligned}
V_{S_{\text {s }+1}}-V_{S_{\text {a }}} & =g\left(\frac{S_{n+1}}{S^{*}}\right)-g\left(\frac{S_{n}}{S^{*}}\right) \\
& =\frac{S_{n+1}-S_{n}}{S^{*}}-\ln S_{n+1}+\ln S_{n} \\
& =\left(\frac{1}{S^{*}}-\frac{\ln S_{n+1}-\ln S_{n}}{S_{n+1}-S_{n}}\right)\left(S_{n+1}-S_{n}\right) \\
& =\frac{1}{S^{*}}\left(1-S^{*} \frac{\ln S_{n+1}-\ln S_{n}}{S_{n+1}-S_{n}}\right)\left(S_{n+1}-S_{n}\right) .
\end{aligned}
$$

By using the mean value theorem we have:

$$
1-S^{*} \frac{\ln S_{n+1}-\ln S_{n}}{S_{n+1}-S_{n}} \leq 1-\frac{S^{*}}{S_{n+1}}
$$

Hence,

Now, let us put

$$
\begin{aligned}
V_{S_{\mathrm{w}+1}}-V_{S_{\mathrm{a}}} & \leq \frac{1}{S^{*}}\left(1-\frac{S^{*}}{S_{n+1}}\right)\left(S_{n+1}-S_{n}\right) \\
& \leq \frac{h}{S^{*}}\left(1-\frac{S^{*}}{S_{n+1}}\right)\left(B-\mu_{1} S_{n+1}-f\left(S_{n+1}, I_{n+1}^{j}\right)\right) \\
& \leq \frac{h}{S^{*}}\left(1-\frac{S^{*}}{S_{n+1}}\right)\left(\mu_{1} S^{*}+f\left(S^{*}, I^{*}\right)-\mu_{1} S_{n+1}-f\left(S_{n+1}, I_{n+1}^{j}\right)\right) \\
V_{S_{\mathrm{w}+1}}-V_{S} \leq & \frac{h}{S^{*}}\left(1-\frac{S^{*}}{S_{n+1}}\right)\left(\mu_{1} S^{*}-\mu_{1} S_{n+1}+f\left(S^{*}, I^{*}\right)-f\left(S_{n+1}, I_{n+1}^{j}\right)\right) \\
\leq & -\frac{h \mu_{1}}{S^{*}} \frac{\left(S_{n+1}-S^{*}\right)^{2}}{S_{n+1}}+\frac{h}{S^{*}}\left(1-\frac{S^{*}}{S_{n+1}}\right)\left(f\left(S^{*}, I^{*}\right)-f\left(S_{n+1}, I_{n+1}^{j}\right)\right) \\
\leq & -\frac{h \mu_{1}}{S^{*}} \frac{\left(S_{n+1}-S^{*}\right)^{2}}{S_{n+1}}+\frac{h f\left(S^{*}, I^{*}\right)}{S^{*}}\left(1-\frac{S^{*}}{S_{n+1}}\right)\left(1-\frac{f\left(S_{n+1}, I_{n+1}^{j}\right)}{f\left(S^{*}, I^{*}\right)}\right) .
\end{aligned}
$$

and

we have,

$$
\begin{gathered}
x_{n+1}=\frac{S_{n+1}}{S^{*}}, y_{n+1}=\frac{I_{n+1}}{I^{*}}, z_{n+1}^{j}=\frac{I_{n+1}^{j}}{I^{*}} . \\
F\left(z_{n+1}^{j}\right)=\frac{f\left(S_{n+1}, I^{*} z_{n+1}^{j}\right)}{f\left(S^{*}, I^{*}\right)}=\frac{f\left(S_{n+1}, I_{n+1}^{j}\right)}{f\left(S^{*}, I^{*}\right)}
\end{gathered}
$$

$$
\xi=\max \left\{\frac{h f\left(S^{*}, I^{*}\right)}{S^{*}}, \frac{h f\left(S^{*}, I^{*}\right)}{I^{*}}, 1\right\}
$$

$$
\begin{aligned}
V_{S_{\text {n+1 }}}-V_{S_{\text {a }}} & \leq-\frac{h \mu_{1}}{S^{*}} \frac{\left(S_{n+1}-S^{*}\right)^{2}}{S_{n+1}}+\frac{h f\left(S^{*}, I^{*}\right)}{S^{*}}\left(1-\frac{S^{*}}{S_{n+1}}\right)\left(1-\frac{f\left(S_{n+1}, I_{n+1}^{j}\right)}{f\left(S^{*}, I^{*}\right)}\right) \\
& \leq-\frac{h \mu_{1}}{S^{*}} \frac{\left(S_{n+1}-S^{*}\right)^{2}}{S_{n+1}}+\frac{h f\left(S^{*}, I^{*}\right)}{S^{*}}\left(1-\frac{1}{x_{n+1}}\right)\left(1-F\left(z_{n+1}^{j}\right)\right) \\
& \leq-\frac{h \mu_{1}}{S^{*}} \frac{\left(S_{n+1}-S^{*}\right)^{2}}{S_{n+1}}+\frac{h f\left(S^{*}, I^{*}\right)}{S^{*}}\left(1-F\left(z_{n+1}^{j}\right)-\frac{1}{x_{n+1}}+\frac{F\left(z_{n+1}^{j}\right)}{x_{n+1}}\right) \\
& \leq-\frac{h \mu_{1}}{S^{*}} \frac{\left(S_{n+1}-S^{*}\right)^{2}}{S_{n+1}}+\xi\left(1-F\left(z_{n+1}^{j}\right)-\frac{1}{x_{n+1}}+\frac{F\left(z_{n+1}^{j}\right)}{x_{n+1}}\right)
\end{aligned}
$$


Now, let us calculate $V_{I_{m+1}}-V_{I_{\mathrm{m}}}$. We use the mean value theorem and we assume that $I_{n} \leq I_{n+1}$. Notice that the computation is the same when $I_{n+1} \leq I_{n}$.

$$
\begin{aligned}
V_{I^{m+1}}-V_{I_{\text {m }}} & =g\left(\frac{I_{n+1}}{I^{*}}\right)-g\left(\frac{I_{n}}{I^{*}}\right) \\
& =\frac{I_{n+1}-I_{n}}{I^{*}}-\ln I_{n+1}+\ln I_{n} \\
& =\left(\frac{1}{I^{*}}-\frac{\ln I_{n+1}-\ln I_{n}}{I n+1-I_{n}}\right)\left(I_{n+1}-I_{n}\right) \\
& =\frac{1}{I^{*}}\left(1-I^{*} \frac{\ln I_{n+1}-\ln I_{n}}{I_{n+1}-I_{n}}\right)\left(I_{n+1}-I_{n}\right) .
\end{aligned}
$$

By using the mean value theorem, we have:

$1-I^{*} \frac{\ln I_{n+1}-\ln I_{n}}{I_{n+1}-I_{n}} \leq 1-\frac{I^{*}}{I_{n+1}}$

Therefore,

$$
\begin{aligned}
V_{I+1}-V_{I^{*}} & \leq \frac{h}{I^{*}}\left(1-\frac{I^{*}}{I_{n+1}}\right)\left(f\left(S_{n+1}, I_{n+1}^{j}\right)-\left(\mu_{2}+\gamma\right) I_{n+1}\right) \\
& \leq \frac{h f\left(S^{*}, I^{*}\right)}{I^{*}}\left(1-\frac{I^{*}}{I_{n+1}}\right)\left(\frac{f\left(S_{n+1}, I_{n+1}^{j}\right)}{f\left(S^{*}, I^{*}\right)}-\frac{\left(\mu_{2}+\gamma\right) I_{n+1}}{f\left(S^{*}, I^{*}\right)}\right) \\
& \leq \frac{h f\left(S^{*}, I^{*}\right)}{I^{*}}\left(1-\frac{I^{*}}{I_{n+1}}\right)\left(\frac{f\left(S_{n+1}, I_{n+1}^{j}\right)}{f\left(S^{*}, I^{*}\right)}-\frac{I_{n+1}}{I^{*}}\right) \\
& \leq \frac{h f\left(S^{*}, I^{*}\right)}{I^{*}}\left(1+\frac{f\left(S_{n+1}, I_{n+1}^{j}\right)}{f\left(S^{*}, I^{*}\right)}-\frac{I_{n+1}}{I^{*}}-\frac{I^{*}}{I_{n+1}} \frac{f\left(S_{n+1}, I_{n+1}^{j}\right)}{f\left(S^{*}, I^{*}\right)}\right) \\
& \leq \frac{h f\left(S^{*}, I^{*}\right)}{I^{*}}\left(1+F\left(z_{n+1}^{j}\right)-y_{n+1}-\frac{F\left(z_{n+1}^{j}\right)}{y_{n+1}}\right) \\
& \leq \xi\left(1+F\left(z_{n+1}^{j}\right)-y_{n+1}-\frac{F\left(z_{n+1}^{j}\right)}{y_{n+1}}\right) .
\end{aligned}
$$

Now, let us evaluate $V_{+\mathrm{a}+1}-V_{+_{\mathrm{w}}}$.

$$
\begin{aligned}
V_{+_{\text {w }}+1}-V_{+_{\text {a }}} & =\sum_{l=0}^{j}\left[g\left(\frac{I_{n+1-l}}{I^{*}}\right)-g\left(\frac{I_{n-l}}{I^{*}}\right)\right] \\
& =g\left(z_{n+1}\right)-g\left(z_{n}^{j}\right) \\
& =z_{n+1}-z_{n}^{j}-\ln z_{n+1}+\ln z_{n}^{j} \\
& \leq \xi\left(z_{n+1}-z_{n}^{j}-\ln z_{n+1}+\ln z_{n}^{j}\right) .
\end{aligned}
$$

By adding and subtracting

$$
1+\ln x_{n+1}, \ln \frac{F\left(z_{n+1}^{j}\right)}{x_{n+1}} \text { and } \ln \frac{F\left(z_{n+1}^{j}\right)}{y_{n+1}}
$$

we have: 


$$
\begin{aligned}
& V_{n+1}-V_{n} \leq-\frac{h \mu_{1}}{S^{*}} \frac{\left(S_{n+1}-S^{*}\right)^{2}}{S_{n+1}}+\xi\left(-z_{n}^{j}+1+\ln z_{n}^{j}+1-\ln y_{n+1}-\frac{1}{x_{n+1}}\right. \\
& +\ln x_{n+1}+1-1+\ln \frac{1}{x_{n+1}}+\frac{F\left(z_{n+1}^{j}\right)}{x_{n+1}}+\ln \frac{F\left(z_{n+1}^{j}\right)}{x_{n+1}}-\ln \frac{F\left(z_{n+1}^{j}\right)}{x_{n+1}} \\
& \left.-\frac{F\left(z_{n+1}^{j}\right)}{y_{n+1}}+\ln \frac{F\left(z_{n+1}^{j}\right)}{y_{n+1}}-\ln \frac{F\left(z_{n+1}^{j}\right)}{y_{n+1}}\right) \\
& \leq-\frac{h \mu_{1}}{S^{*}} \frac{\left(S_{n+1}-S^{*}\right)^{2}}{S_{n+1}}+\xi\left(-z_{n}^{j}+1+\ln z_{n}^{j}-\frac{1}{x_{n+1}}+1+\ln \frac{1}{x_{n+1}}\right. \\
& \left.+\frac{F\left(z_{n+1}^{j}\right)}{x_{n+1}}-1-\ln \frac{F\left(z_{n+1}^{j}\right)}{x_{n+1}}-\frac{F\left(z_{n+1}^{j}\right)}{y_{n+1}}+1+\ln \frac{F\left(z_{n+1}^{j}\right)}{y_{n+1}}\right) \\
& \leq-\frac{h \mu_{1}}{S^{*}} \frac{\left(S_{n+1}-S^{*}\right)^{2}}{S_{n+1}} \\
& +\xi\left[-g\left(z_{n}^{j}\right)-g\left(\frac{1}{x_{n+1}}\right)+g\left(\frac{F\left(z_{n+1}^{j}\right)}{x_{n+1}}\right)-g\left(\frac{F\left(z_{n+1}^{j}\right)}{y_{n+1}}\right)\right] .
\end{aligned}
$$

By using $\mathbf{H 4}$ and the fact that the function $g$ is monotone increasing on each side of point 1 and is minimized at this point 1 , we obtain:

$$
g\left(\frac{F\left(z_{n+1}^{j}\right)}{x_{n+1}}\right) \leq g\left(\frac{F\left(z_{n+1}^{j}\right)}{y_{n+1}}\right) .
$$

Thus, we have

$$
V_{n+1}-V_{n} \leq 0
$$

Hence, by the Lyapunovs theorems on the global asymptotical stability for difference equations [7], we obtain that the endemic equilibrium $E^{*}$ is globally asymptotically stable.

\section{Simulation and comments}

In this section, we present a numerical simulation of continuous-time model and discrete one given respectively by models (1) and (3). Notice that Scilab is the software used for the simulations. We give the representation from the value of our step size $h$ and the reproduction rate $R_{0}$. We discuss when reproduction rate $R_{0} \leq 1$ and $R_{0}>1$. The black curve gives the evolution of the different class for the continuous time model and the red curve the evolution of the different class for the discrete time model. In our, simulation we use the mass action incidence rate function defined by $f(S, I)=\beta S I$ where $\beta$ is a nonnegative constant. The parameters used in the simulation are: $B=$ $100 ; \mu_{1}=0.1 ; \mu_{2}=0.02 ; \mu_{3}=0.03 ; \gamma=0.2 ; \beta=0.00023 ; c=0.2$; from this value we have $R_{0}=0.21<1$. When we change the value of $\beta$ to $\beta=0.023$, we get $R_{0}=20.91>1$.

\section{Conclusion}

In this paper, motivate by the fact that discrete models are more appropriate forms than the continuous ones in order to fit the statistical data concerning infectious diseases, we have studied the discrete forward difference version for a SIR model with general incidence function. We use the backward Euler discretization with the step size $h$; the reproduction rate $R_{0}$ is obtained and we show that the disease-free equilibrium $E_{0}$ is globally asymptotically stable if $R_{0} \leq 1$ and the unique endemic equilibrium $E^{*}$ exist and is globally asymptotically stable if $R_{0}>1$ for this discrete SIR epidemic model with general incidence function (3) by using the technique of Lyapunov functional. We observe that, there is no more great difference between the two models, the dynamics are almost the same. The step size $h$ is $h=1$, but this step can be seen as a bifurcation parameter and we project to investigate this in our future works.

\section{Competing interests}

The authors declare that they have no competing interests.

\section{Funding}

Not applicable.

\section{Author's contribution}

Aboudramane Guiro provide the subject, wrote the introduction and the conclusion and verified some calculation. Dramane Ouedraogo conceived the study and computed the equilibria and their local stabilities. Harouna Ouedraogo wrote mathematical formula, bring up the Lyapunov functional and did all the calculus with the other authors. All the authors read and approved the final manuscript. 
The authors want to thank the anonymous referee for his valuable comments on the paper.

\section{References}

[1] C. Connell McCuskey, Global stability for an SEIR epidemiological with varying infectivity and infinite delay, Math. Biosci. Eng. 6 (2009) 603-610.

[2] C. Connell McCuskey, Global stability for an SIR epidemic model with delay and nonlinear incidence, Nonlinear Anal. RWA 10(2009) 3175-3189.

[3] C. Connell McCuskey, Complete global stability for an SIR epidemic model with delay distributed or discrete, Nonlinear Anal. RWA 11(2010) 55-59.

[4] Y. Enatsu, Y. Nakata, Y. Muroya, Global stability for class of discrete SIR epidemic models, Mathematical Biosciences and Engineering 7 (2010) 347 361.

[5] A. Guiro, D. Ngom, D. Ouedraogo, Stability analysis for a class of discrete Schistosomiasis models with general incidence, Advances in Difference Equation, (2017), $1-16$.

[6] A. Guiro, D. Ouédraogo, H. Ouedraogo, Global stability for a delay SIR epidemic model with general incidence function, observers design, Submitted to Applied mathematics.

[7] J. P. Lasalle, The stability of Dynamical Systems, SIAM, Philadelphia, 1976.

[8] V. Lakshmikantham, S. Leela, A. A. Martynyuk, Stability Analysis of Nonlinear Systems, Marcel Dekker, New York, 1989.

[9] Z. Teng, Y. Wang, M. Rehim, On the backward difference scheme for a class of SIRS epidemic models with nonlinear incidence. J. Computation Analysis and Applications, vol. 20, No 7, 2016, pp. (1268-1289).

[10] P. Van den Driesche and J. Watmough, Reproduction Numbers and Substhreshold Endemic Equilibria for the compartmental Model of Disease Transmission, Mathematical Biosciences, 180(2002) No.1 - 2, pp.29-48.

[11] M. Sekiguchi, E. Ishiwata, Global dynamics of a discrete SIRS epidemic model with time delay, J. Math. Anal. Appl., 371, 195 - 202(2010).

[12] M. Sekiguchi, Permanence of a discrete SIRS epidemic model with time delays, Appl. Math. Letters, 23, 1280 - 1285(2010).

[13] Z. Hu, Z. Teng, H. Jiang, Stability analysis in a class of discrete SIRS epidemic models, Nonlinear Anal.: RWA, 13, 2017 - 2033(2012).

[14] R. Xu, Z. Ma, Global stability of a SIR epidemic model with nonlinear incidence rate and time delay, Nonlinear Anal. RWA 10(2009)3175 - 3189.

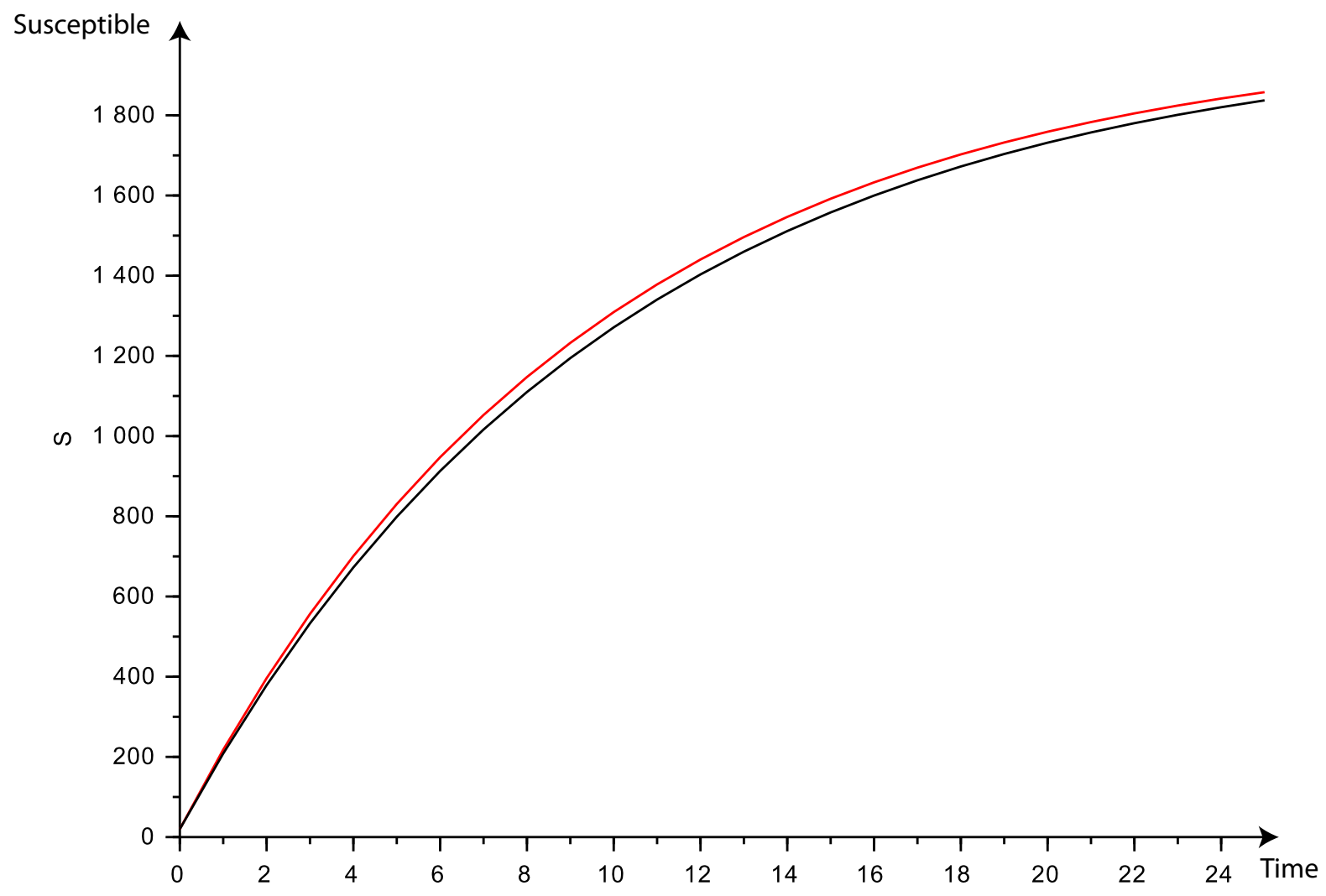

Figure 1: Susceptible: Case where $R_{0}<1$, Red curve represents the discrete model and black one the continuous model. 


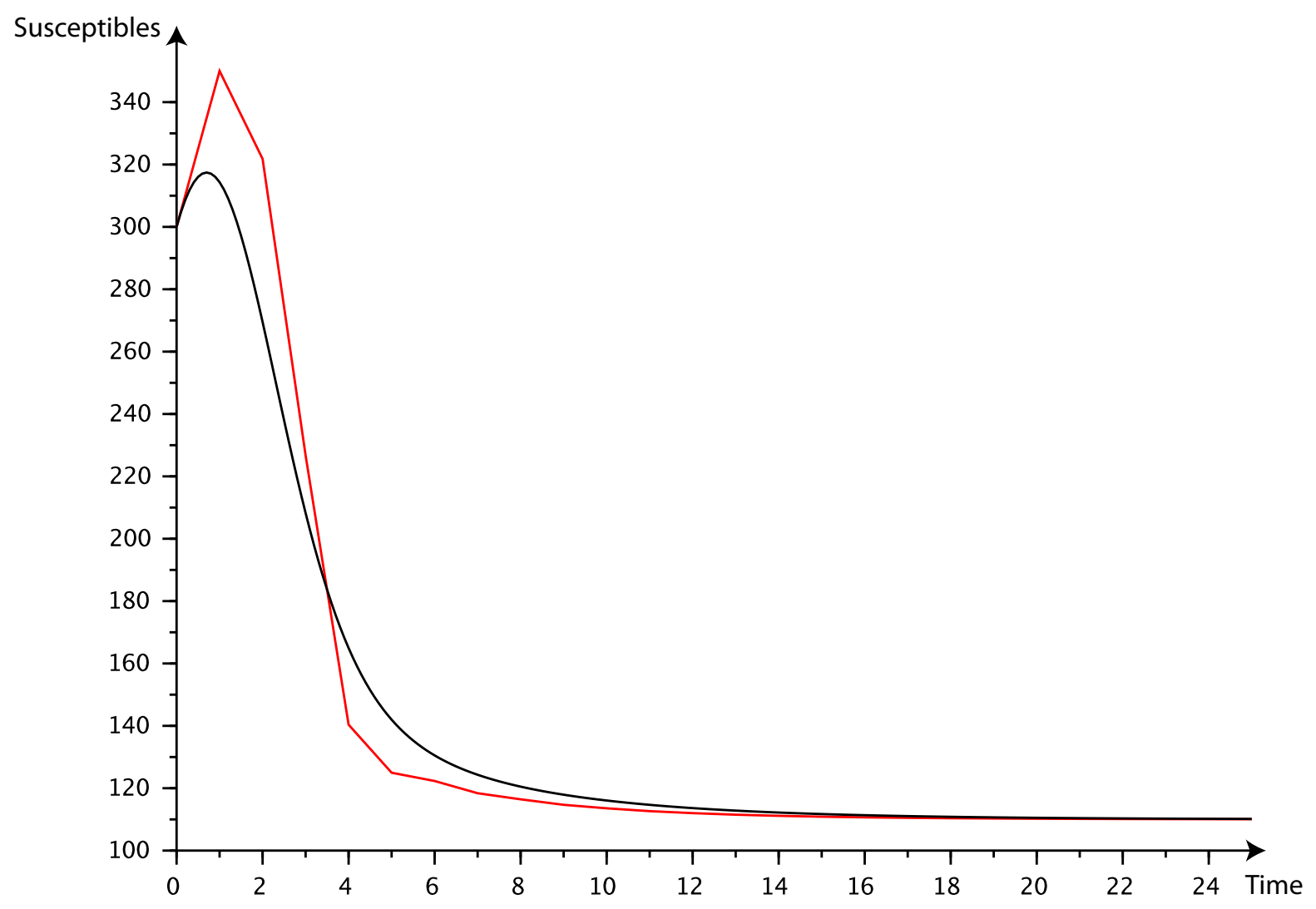

Figure 2: Susceptible: Case where $R_{0}>1$, Red curve represents the discrete model and black one the continuous model. 


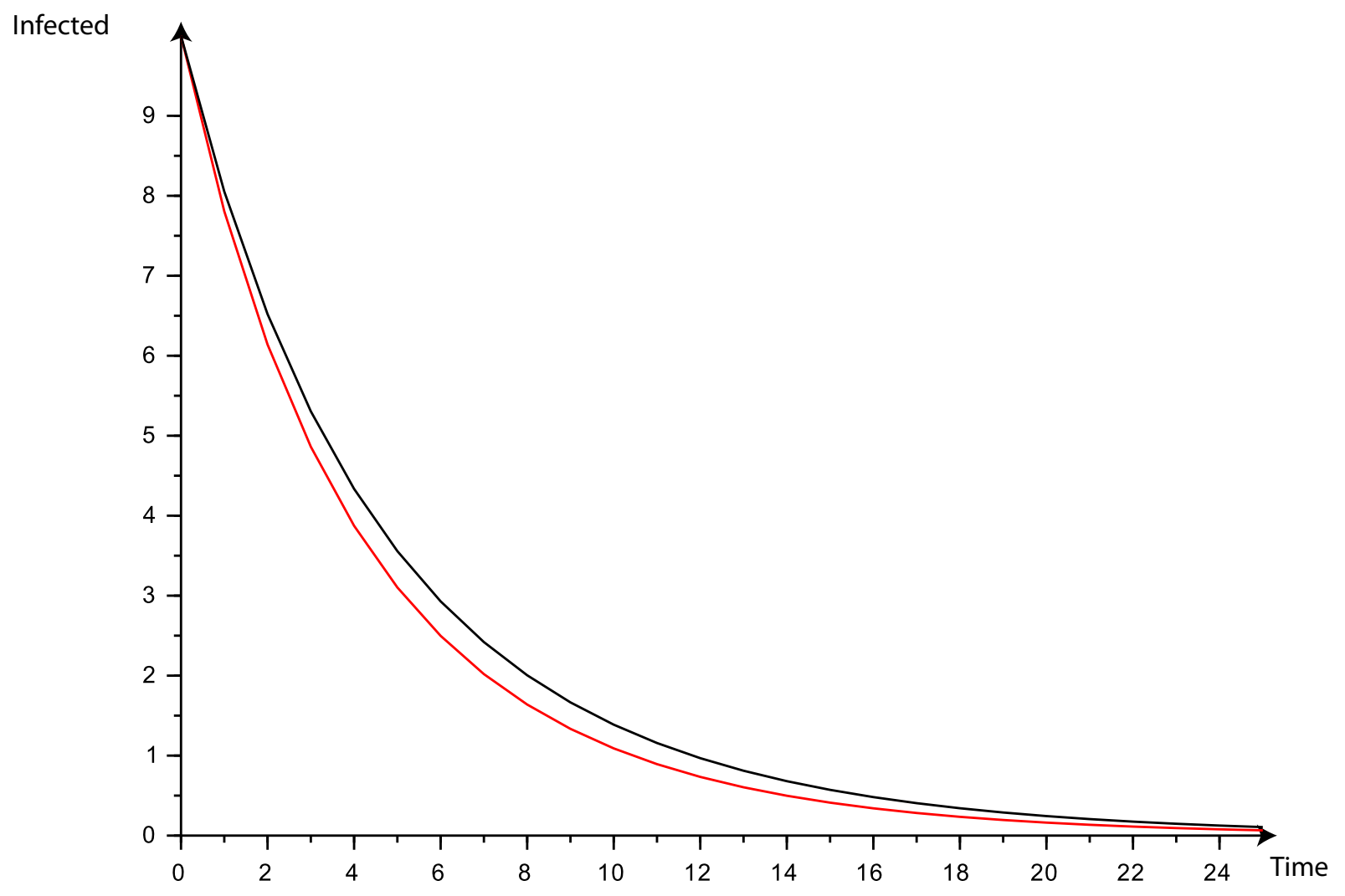

Figure 3: Infected: Case where $R_{0}<1$, Red curve represents the discrete model and black one the continuous model. 


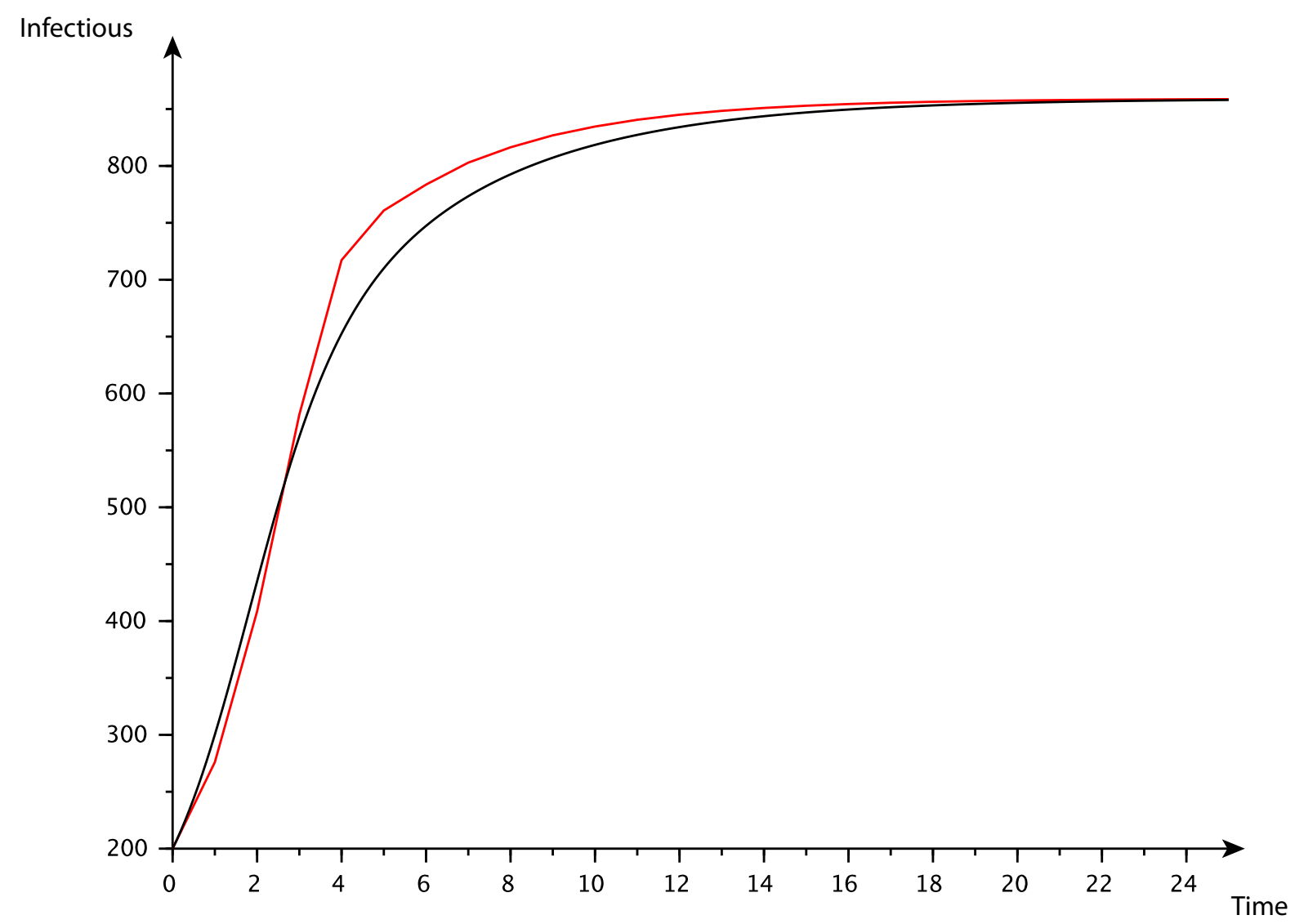

Figure 4: Infected: Case where $R_{0}>1$, Red curve represents the discrete model and black one the continuous model. 


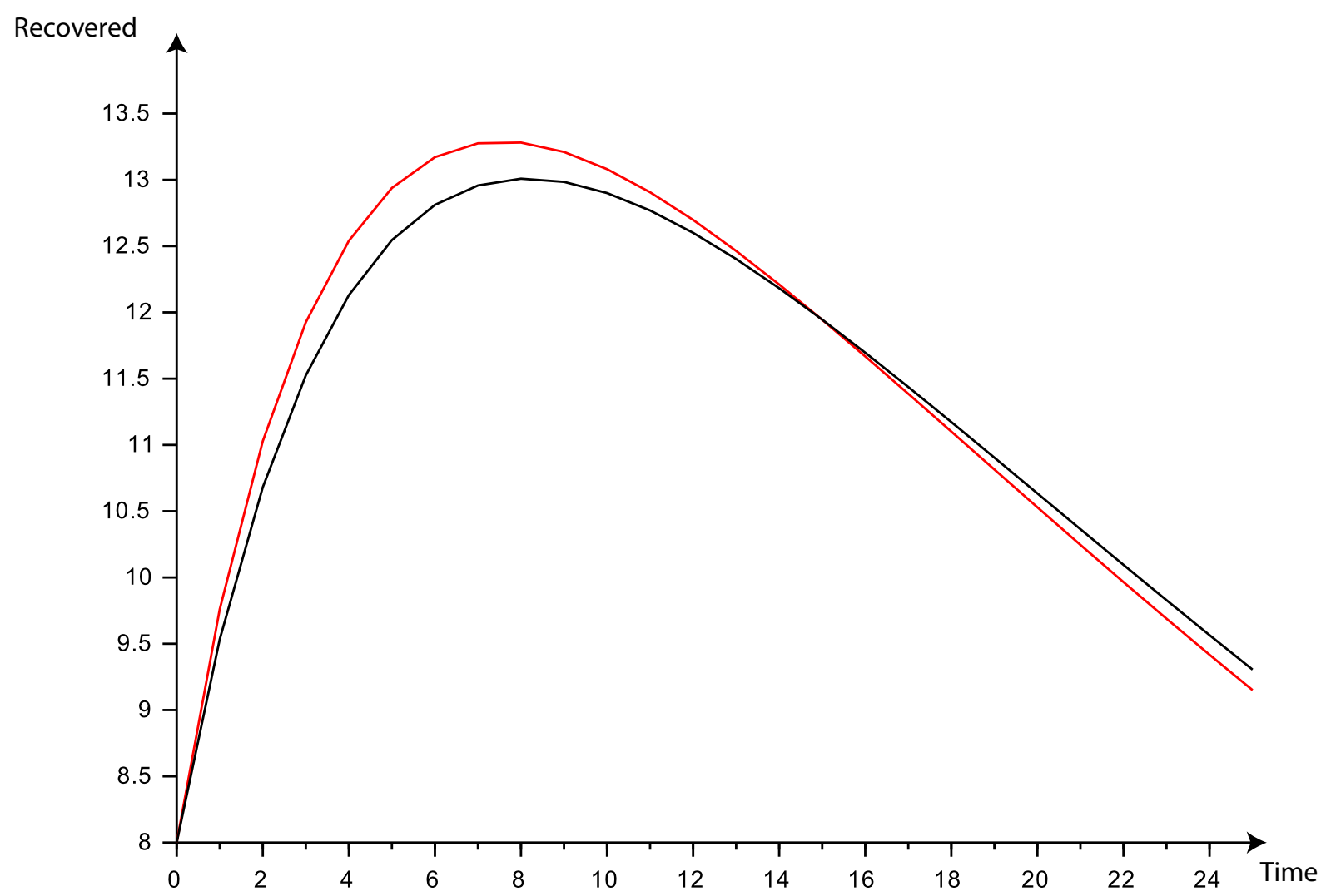

Figure 5: Recovered: Case where $R_{0}<1$, Red curve represents the discrete model and black one the continuous model. 


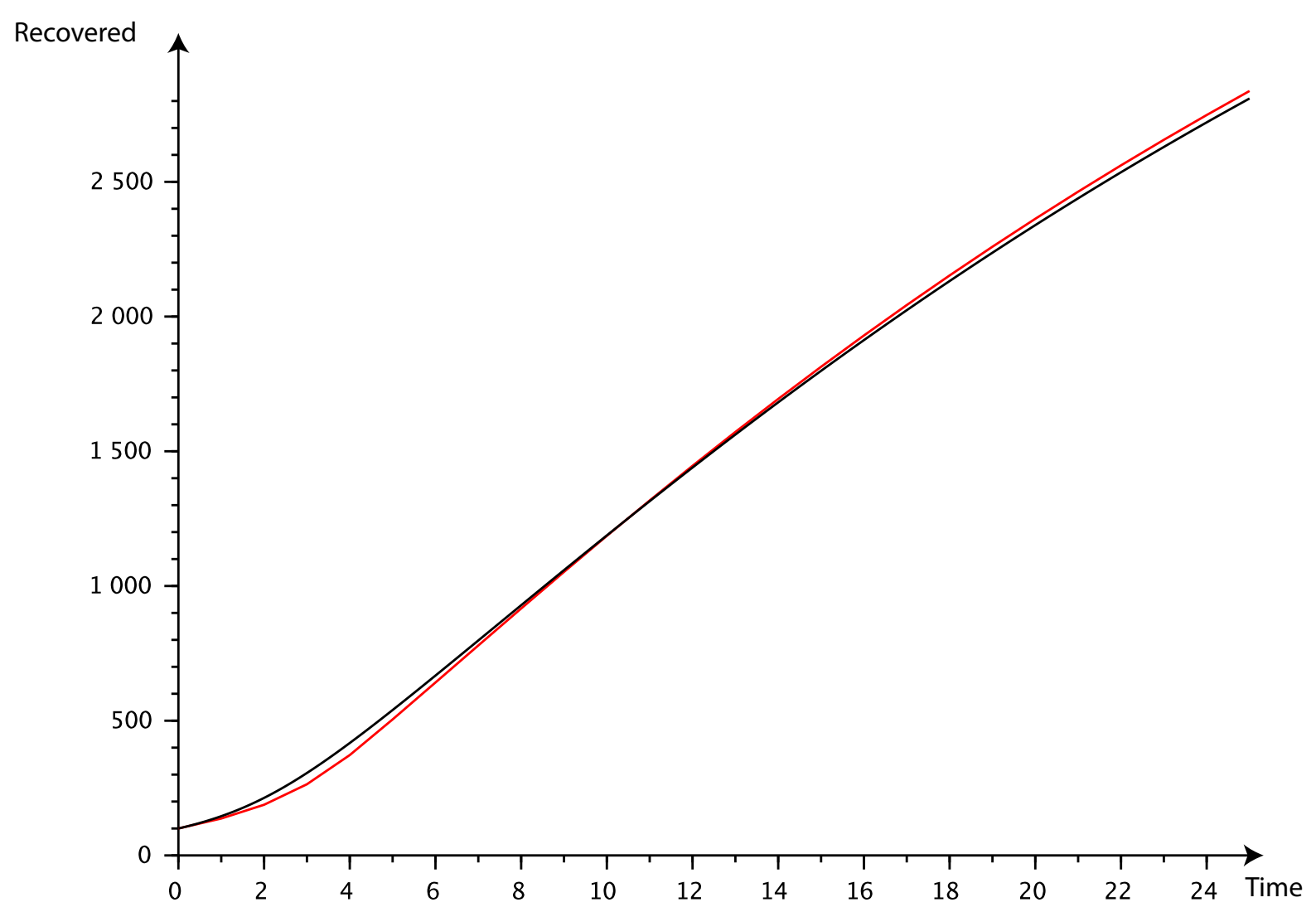

Figure 6: Recovered: Case where $R_{0}>1$, Red curve represents the discrete model and black one the continuous model. 